\title{
Visual Contrast Enhancement by Histogram Modification Based on Generalized Extreme Value Distribution
}

\author{
Preecha Vonghirandecha ${ }^{1}$, Montri Karnjanadecha ${ }^{1}$ and Sathit Intajag ${ }^{2, *}$ \\ ${ }^{1}$ Department of Computer Engineering, Faculty of Engineering \\ ${ }^{2}$ Department of Computer Science, Faculty of Science \\ Prince of Songkla University, Hat Yai, Songkhla, Thailand. \\ *Corresponding Author:sathit.i@psu.ac.th
}

\begin{abstract}
Contrast enhancement is a crucial method for improving the quality of an image. This paper proposes a visual contrast enhancement of a color image by using histogram modification. Transfer function of the modified histogram was designed with generalized extreme value distribution. To automatically enhance image contrast and tone meanwhile improving color balance, three parameters of the distribution that consists of shape, scale, and location were estimated by probability weighted moments. The location parameter was employed to improve brightness and color balance. The scale and shape was provided to enhance contrast and to adjust tone of the images. The proposed algorithm was evaluated with a low dynamic range (true-color images) and a high dynamic range images.

Keywords: Histogram modification, Generalized extreme value distribution, Visual contrast enhancement, Color balance, Tone mapping.
\end{abstract}

\section{Introduction}

Due to the widespread applications of digital cameras, many digital color images have been taken under improper lighting conditions as a result of low visual quality. To solve this problem, recently many proposed methods have been proposed to produce the better quality images. Contrast enhancement based on histogram modification (HM) $)^{(1-3)}$ is a widely used technique for improving visual quality of the low contrast pictures in an image processing.

Histogram equalization (HE) is normally used to enhance the brightness and contrast of an image by using cumulative distribution function for stretching its dynamic range. HE is often questioned for excessive enhancement; because, it may shift the mean intensity values to the middle gray level of the intensity range. To overcome this mean-shift problem, many researchers ${ }^{(4-9)}$ have proposed methods to solve the mean shift problem of HE. Fundamental objective of the solving mean shift techniques provides the output images with the mean of brightness close to the original. Those techniques are not suit for underexpose or overexpose images; because, they will lead to human visual perception loss problem.

Recently, visual contrast enhancement algorithm (VCEA) based on histogram equalization was introduced by Meng-Liang Chung et al. ${ }^{(10)}$. The image results of VCEA have much more visual quality than other HE-based methods; however, the space adjustment mechanism cannot control the brightness mean. This mechanism will affect to improper appearance of color balance.

In this paper, a new visual contrast enhancement is proposed. Our model employs histogram modification ${ }^{(2)}$ with the generalized extreme value (GEV) distribution ${ }^{(11)}$ to characterize the transfer functions. Three parameters of GEV, which consist of shape, scale, and location, are estimated by probability weighted moments (PWMs) ${ }^{(12)}$.

By setting optimal values of the GEV parameters can use to enhance the retinal images ${ }^{(13)}$; however, our proposed algorithm uses the standard white point, D55, to specify location parameters for controlling the brightness and color balance. The scale and shape parameters are employed to improve contrast and to adjust tone of color images, respectively. Our algorithm employs Kullback-Leibler divergence $(\mathrm{KLD})^{(14)}$ to select estimated contrast and tone parameter values. These parameters are manipulated by scale and shape parameters. Optimal tuning process of the three parameters is employed to formulate the transfer functions of HM and thus the algorithm can produce good 
quality color images.

The performance of the proposed scheme is evaluated with public available datasets True-color Kodak Dataset ${ }^{(15)}$ (for low dynamic range images) and ESPL-LIVE HDR Image Quality Dataset ${ }^{(16)}$.

The paper proceeds as follows: Section 2 describes designing transfer functions, which consists of histogram modification, GEV distribution, and PWMs estimation; Section 3 describes the proposed algorithm; Section 4 presents the experimental results and the paper concludes in Section 5 .

\section{Transfer Functions}

The proposed method relates to three topics for fabricating our algorithm. The theme is the transfer functions of histogram modification. The either two topics are provided to support the HM that is a GEV distribution and its parameter estimation method by PWMs.

\subsection{Histogram Modification}

Histogram analysis dates back to the early era of digital image processing ${ }^{(1-3)}$. It provides to analyze an intensity distribution for characterizing and designing to enhance pictures. As it includes a process for analyzing characteristics and design, the histogram has been named a histogram equalization, histogram matching, histogram specification, or histogram modification. Histogram processing employs mathematical models known as probability distribution functions (PDF). A simple version of histogram equalization uses a uniform distribution. The advance processes need to specify the shape of frequency distributions. The PDF has to support the advance processes.

Let $x$ be the intensity levels of an image, $X$, in the range $[0, L-1]$ (where $L$ denotes the maximum intensity value). The number of pixels in each intensity level, $h(x)$, is histogram of the image, $X$. $h(x)$ could also be expressed as a percentage of the pixel numbers against the total number of pixels in the image, $X$; that is, $p(x)=h(x) / M N$, where the size of image is $M \times N$ pixels. In statistical terms, $p(x)$ is the PDF.

Histogram matching based on $\mathrm{HM}^{(2)}$ could be regarded as a monotonic point transformation, $g_{d}=T\left\{f_{c}\right\}$. The input variable, $f_{c} \in\left[f_{1}, f_{C}\right]$, was mapped into an output variable, $g_{d} \in\left[g_{1}, g_{D}\right]$, such that the output probability distribution, $P_{R}\left\{g_{d}=b_{d}\right\}$, follows some desired form for a given input probability distribution, $P_{R}\left\{f_{c}=a_{c}\right\} . a_{c}$ and $b_{d}$ represent reconstruction values between the $c^{\text {th }}$ and $d^{\text {th }}$ intensity levels. $C$ and $D$ were the maximum intensity values of the histogram; thus, the sum of input and output probability distribution must be equal to unity:

$$
\begin{aligned}
& \sum_{c=1}^{C} P_{R}\left\{f_{c}=a_{c}\right\}=1 \\
& \sum_{d=1}^{D} P_{R}\left\{g_{d}=b_{d}\right\}=1 .
\end{aligned}
$$

The probability that pixels of the input image had an amplitude less than or equal to $a_{c}$ must be equal to the probability that pixels of the output image have amplitude less than or equal to $b_{d}$, where $b_{d}=T\left\{a_{c}\right\}$ because the transformation is monotonic. Hence

$$
\sum_{n=1}^{d} P_{R}\left\{g_{n}=b_{n}\right\}=\sum_{m=1}^{c} P_{R}\left\{f_{m}=a_{m}\right\} .
$$

The histogram modification in (3) can be obtained in approximate form by replacing the discrete probability distributions of (2) by continuous probability densities.

$$
\int_{g_{\min }}^{g} p_{g}(g) d g=\int_{f_{\min }}^{f} p_{f}(f) d f
$$

where $p_{f}(f)$ and $p_{f}(g)$ are the probability densities of $f$ and $g$, respectively. From the given image, $X$, the integral on the right was replaced by the cumulative distribution function $(\mathrm{CDF}), P_{f}(x)=\sum_{x=f_{\min }}^{f} p(x)$. Thus, equation (3) was in the form

$$
\int_{g_{\min }}^{g} p_{g}(g) d g=P_{f}(x) .
$$

The histogram was modified by many probability functions such as uniform, hyperbolic, exponential, and Rayleigh distribution, etc. ${ }^{(2)}$. Those distributions usually control only the range (uniform and hyperbolic) and some of them can adjust the shape parameters (exponential and Rayleigh). In a color image enhancement, the process needs a location parameter to control the color balance. In this paper, GEV distribution had three parameters consisting of shape, scale, and location, which were provided to modify the histogram of color fundus images ${ }^{(13)}$. 


\subsection{Generalized Extreme Value Distribution}

Frequency analysis was an interesting topic for us because one important topic in a digital image processing has to analyze intensity values; especially, in $\mathrm{HM}^{(2)}$. For a color image, histogram transfer functions need more parameters to enhance the image; therefore, three parameters of GEV could be provided to adjust contrast, brightness, and color balance of the color images. The CDF of a $\mathrm{GEV}^{(11)}$ was given by

$$
F(x)=\left\{\begin{array}{l}
\exp \left\{-\left[1-\kappa \frac{(x-\mu)}{\sigma}\right]^{1 / \kappa}\right\}, \kappa \neq 0,\left\{\begin{array}{c}
\mu+\sigma / \kappa \leq x<\infty, \text { for } \kappa<0 \\
-\infty<x \leq \mu+\sigma / \kappa, \text { for } \kappa>0
\end{array}\right. \\
\exp \left\{-\exp \left[-\frac{(x-\mu)}{\sigma}\right]\right\}, \quad-\infty<x<\infty, \text { for } \kappa=0
\end{array}\right.
$$

where $\mu, \sigma$, and $\kappa$, denote the location, scale, and shape parameters, respectively. When $\kappa=0, \mathrm{CDF}$ is Gumble's type I $(-\infty<x<\infty)$. When $\kappa \neq 0$, CDF includes two types. When $\kappa<$ 0 it becomes Frechet's type II, but when $\kappa>0$ it is the Weibull or type III distribution.

The PDF corresponding to (5) is

$$
p(x)=\left\{\begin{array}{c}
\exp \left\{-(1+\kappa(x-\mu) / \sigma)^{-1 / \kappa}\right\} \frac{1}{\sigma}\left[1+\kappa\left(\frac{x-\mu}{\sigma}\right)\right]^{-\frac{1}{\kappa}-1}, \\
\left\{\begin{array}{c}
\mu+\sigma / \kappa \leq x<\infty, \text { for } \kappa<0 ; \\
-\infty<x \leq \mu+\sigma / \kappa, \text { for } \kappa>0 ;
\end{array}\right. \\
\exp \left\{-\exp \left[-\frac{(x-\mu)}{\sigma}\right]\right\} \frac{1}{\sigma} \exp \left[-\frac{(x-\mu)}{\sigma}\right] \\
-\infty<x<\infty, \text { for } \kappa=0
\end{array}\right.
$$

The transformed intensity, $g$, could be solved by replacing the density function, $p_{g}(g)$, of (4) with the $p(x)$ of (6) and integral the left, which becomes to the CDF of (5). Thus, the transfer function of HM by GEV distribution becomes

$$
g= \begin{cases}\mu+\frac{\sigma}{\kappa}\left[1-\left(-\ln P_{f}(x)\right)^{\kappa}\right] & \text { for } \kappa \neq 0 \\ \mu-\sigma \ln \left(-\ln P_{f}(x)\right) & \text { for } \kappa=0 .\end{cases}
$$

The modified intensity, $g$, of each color band comes from the CDF of the input image and the GEV parameters, which might be estimated by the method of PWMs.

\subsection{Probability Weighted Moments}

Three parameters of GEV distribution could be estimated by PWMs ${ }^{(11,12)}$ as follows:

$$
\begin{gathered}
\hat{\mu}=b_{0}-\frac{\hat{\sigma}}{\hat{\kappa}}[1-\Gamma(1+\hat{\kappa})] \\
\hat{\sigma}=\frac{\left(2 b_{1}-b_{0}\right) \hat{\kappa}}{\left(1-2^{-\hat{\kappa}}\right) \Gamma(1+\hat{\kappa})} \\
\hat{\kappa}=7.8590 z+2.9554 z^{2}
\end{gathered}
$$

where $z=\left(2 b_{1}-b_{0}\right) /\left(3 b_{2}-b_{0}\right)-\log 2 / \log 3$ and $\Gamma$ denotes a gamma function. The values, $b_{0}, b_{1}$, and $b_{2}$, were calculated by employing an unbiased estimator of the first three PWMs that were given by

$$
\beta_{r}=\mu+\frac{\sigma}{\kappa} \frac{\left[1-(r+1)^{\kappa} \Gamma(1+\kappa)\right]}{r+1} .
$$

The unbiased estimator of $\beta_{r}$ is

$$
b_{r}=\sum_{i=1}^{n} \frac{(i-1)(i-2)(i-3) \ldots(i-r)}{n(n-1)(n-2)(n-3) \ldots(n-r)} x(i), \quad r=0,1,2, \ldots
$$

where $x_{(i)}$ denotes the ordered observations from a sample of size $n$, that is $\left\{x_{(1)} \leq x_{(2)} \leq x_{(3)} \leq \cdots \leq x_{(n)}\right\}$.

The transfer function of HM by using GEV distribution as a transfer function was reviewed. The next section will describe the algorithm to enhance the color images by employing HM for adjusting the color balance, brightness, and contrast.

\section{Color Enhancement Algorithm}

The proposed method employs GEV parameters to adjust brightness, contrast, and color balance of natural images by HM. "Aster" image ${ }^{(17)}$ in Fig. 1(a) was provided to demonstrate how our algorithm operates. The algorithm consists of four steps as shown in Fig. 2. The details of each step are described in the following subsections.

\subsection{Initial Values Setting}

The first step initializes the specified parameters of brightness, contrast, and color balance. Input image was resampled by reducing the size following the eyes adaptation for the best view in the fovea, which could be approximated each luminance over a $1^{\circ}$ diameter solid angle corresponding to a potential foveal fixation point in the scene ${ }^{(18)}$. The brightness parameters, Bright $t_{\text {Red }}$,

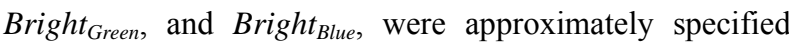
approaching to white point proportion of D55; thus, 
$\left\{\right.$ Bright $_{\text {Red }}$, Bright $_{\text {Green }}$, Bright $\left._{\text {Blue }}\right\}=\{96,100,92\}$.

The scale parameters of each color band using for spread the dynamic ranges of the images were calculated and adapted by the maximum value of the scales from each color band. The tone of the images was iteratively adjusted by the shape parameter.

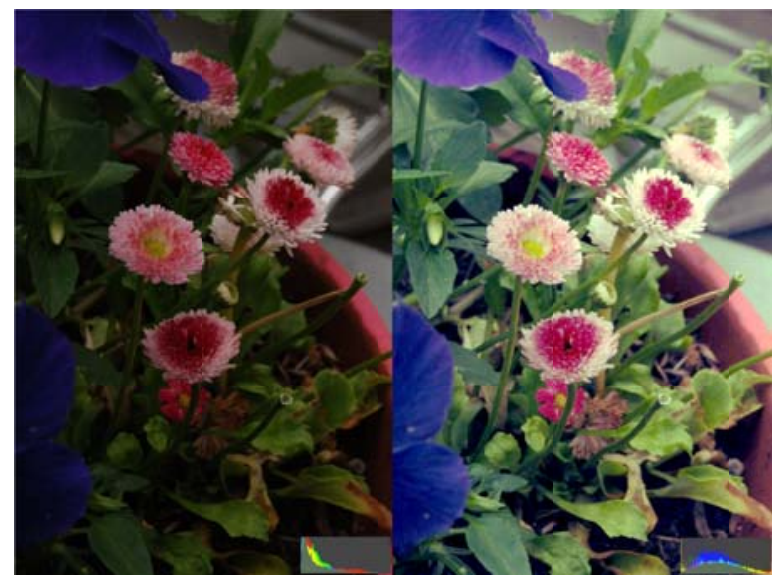

(a) Left: "Aster" dark image (image size: $1071 \times 1600$ pixels, taken by Nikon D3100 ata shutter speed 1/60 and aperture of F/12 $2^{(17)}$ ), Right: Output image,

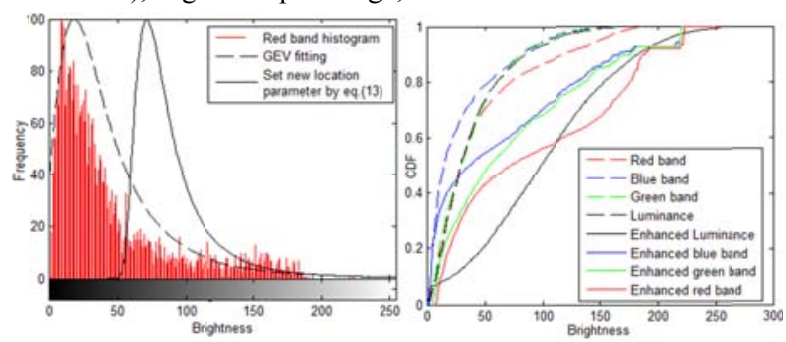

(b) GEV fitting red band,

(c) GEV transfer function

Fig.1. Color image enhancement by tone mapping with generalized extreme value distribution.

\subsection{GEV Parameters Estimation and Design}

The down-sampled image data from the first step was estimated the GEV parameters for each color channel. The three parameters, $\kappa, \sigma$, and $\mu$ were estimated by using PWMs. From the red band of Fig. 1(a), the parameters, shape, scale, and location were approximated to -0.29 , 22.61 , and 24.01 , respectively.

In the designing process, the first moment, $b_{0}=46.06$, represents the mean value of the red band in Fig 1(a), which deviates from the specified brightness value of the red band, Bright $_{\text {Red. }}$. Thus, the new location parameter, $\hat{\mu}^{\prime}$, is recalculated by using (13), where it replaces the first moment, $b_{0}$, in (8) with Bright $_{\text {Red }}$, as given by:

$$
\hat{\mu}^{\prime}=\operatorname{Bright}_{\text {Red }}-\frac{\hat{\sigma}}{\hat{\kappa}}[1-\Gamma(1+\hat{\kappa})] .
$$

However, the new location parameter of the red band, $\hat{\mu}^{\prime}=73.63$, provided the new range, $-4.15<x \leq \infty$, which the intensity of the red band spreads out of range for 8 bits images as seen in Fig. 1(b).

The shape and scale parameters were tuned in the next step for manipulating the image dynamic range. To tune the dynamic range at the same time with improving the image tone, the scale parameter was set to the initialized scale and the shape parameter were evaluated by data boundary.

Let $y$ denote either the lower bound (LB) or the upper bound (UB) of the data; when the boundary condition was found out of range. It was solved by the range of the random variable, $x$, in (6), where Hosking recommends equating $x$ to $\mu+\sigma / \kappa$ and reformulating it for the shape parameter ${ }^{(11)}$. In our case, the shape parameter was resolved by

$$
\hat{\kappa}= \begin{cases}\frac{\ln \left\{\left(2 b_{1}-y\right) /\left(b_{0}-y\right)\right\}}{\ln 2}, & y=\mathrm{LB} \\ \frac{\ln \left\{\left(2 b_{1}+y\right) /\left(b_{0}+y\right)\right\}}{\ln 2}, & y=\mathrm{UB} .\end{cases}
$$

\subsection{Image Tone Tuning}

In our scheme, image tone is enhanced by searching for the optimal shape and scale parameters. The searching range of the shape parameter was determined by evaluating the minimum and maximum boundary values of $\left\{\hat{\kappa}, \hat{\kappa}_{L B}, \hat{\kappa}_{U B}\right\} \quad$ by $\hat{\kappa}_{\min }=\min \left(\hat{\kappa}, \hat{\kappa}_{L B}, \hat{\kappa}_{U B},-0.05\right)$ and $\hat{\kappa}_{\max }=\max \left(\hat{\kappa}, \hat{\kappa}_{L B}, \hat{\kappa}_{U B}, 0.05\right)$, respectively. The interval $\left[\hat{\kappa}_{\text {min }}, \hat{\kappa}_{\text {max }}\right]$ was iterated by incremental value with 0.01 .

In each step for searching the shape parameter, the scale parameter was evaluated by the tolerance value \pm 5 , which provided the fitting dynamic range with the image tone distribution. In each iterative evaluation, the location in (13) and transfer function in (7) were recalculated to update tone distribution, $g$, which was used to generate histograms $H_{x}$ and $H_{T} . H_{x}$ denotes PDF of the output image derived from (7) and $H_{T}$ represents the PDF of the GEV in (6). Both PDFs were evaluated by Kullback-Leibler divergence (KLD) ${ }^{(14)}$ as in the following

$$
\mathrm{KLD}=\sum_{i=1}^{L} \ln \left(H_{T}(i) / H_{X}(i)\right) H_{T}(i)
$$




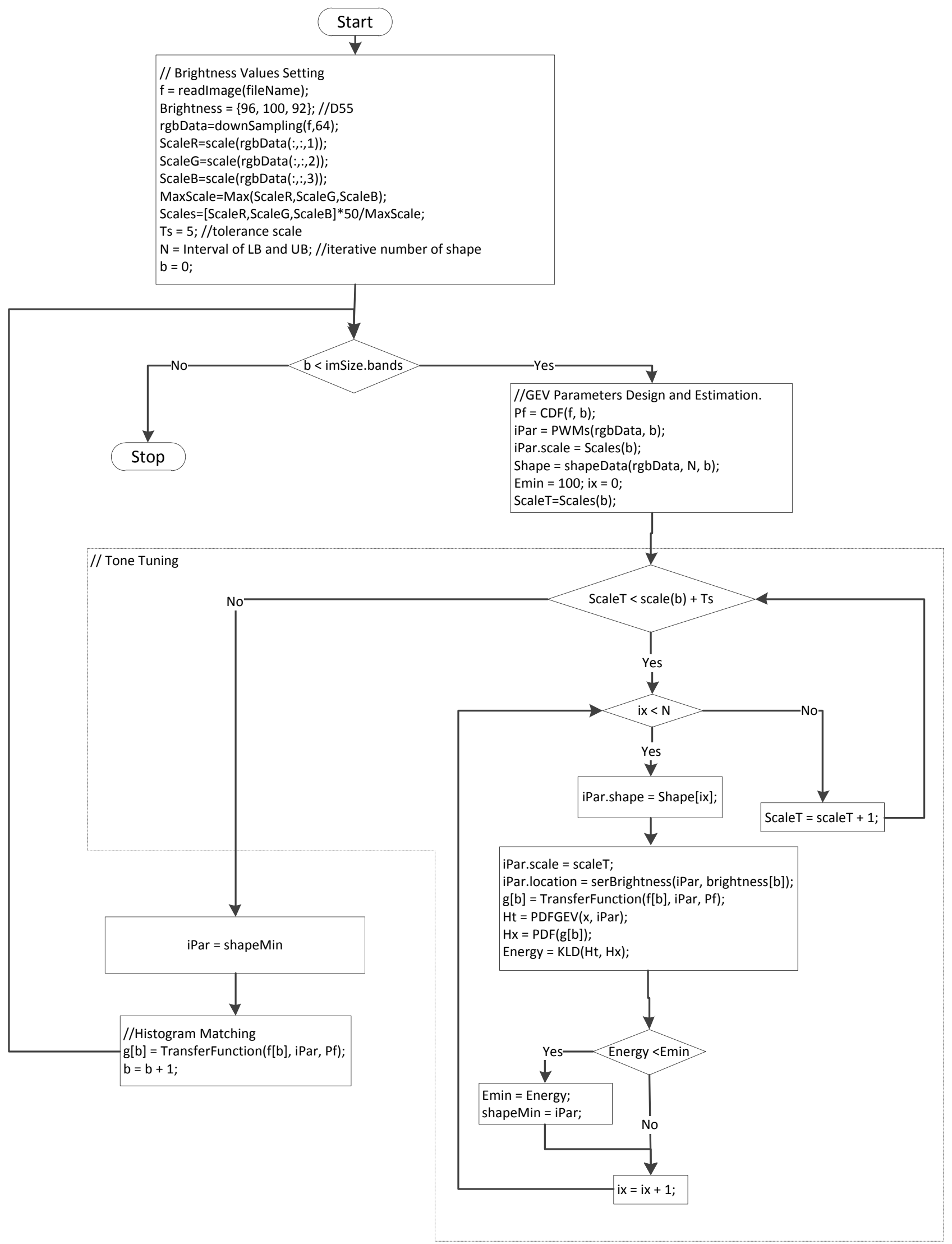

Fig.2.Visual contrast enhancement algorithm by generalized extreme value distribution. 
From evaluation of the GEV parameters, the optimal values were specified by the minimum values of KLD. After completing the iterations, the optimal GEV parameters to modify each color histogram were established.
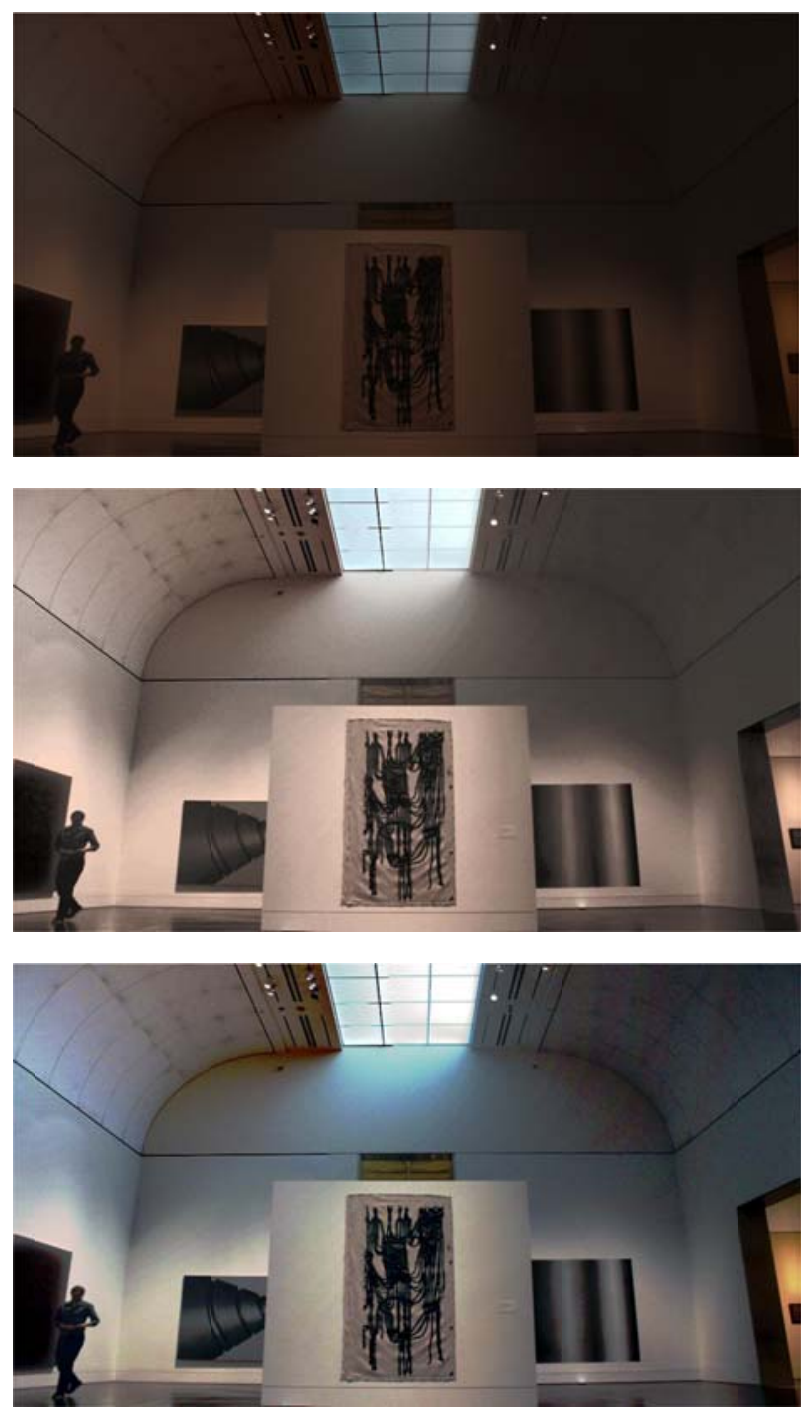

Fig. 3. Blanton Museum image enhancement, input, VCEA, and the proposed method (from top to bottom).

\subsection{Histogram Matching.}

The final step employs the optimal parameters from the step three to adjust the color histograms of each band by the transfer function in (7) as seen in Fig. 1(c). From the red band of Fig. 1(a), the optimal GEV parameters were defined as $\hat{\kappa}=0.50, \hat{\sigma}=75.00$, and $\hat{\mu}^{\prime}=78.59$. The green and blue bands could be operated with the same as the red band as in the demonstration.

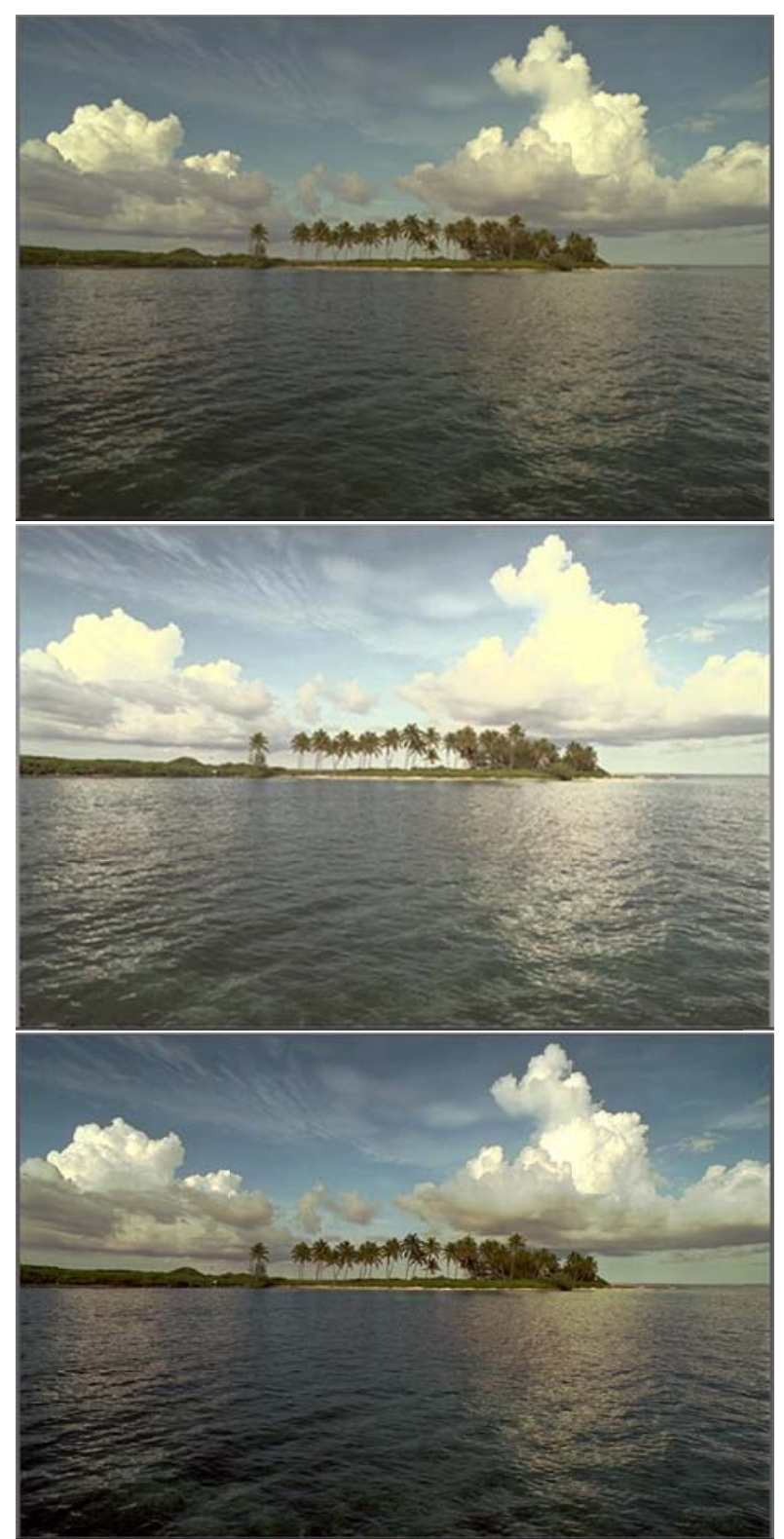

Fig. 4. Sea image enhancement, input, VCEA, and the proposed method (from top to bottom).

Table 1. GEV parameters to specify histogram.

\begin{tabular}{|c|c|l|l|}
\hline Images & Parameter & \multicolumn{1}{|c|}{ Input image (R,G,B) } & Output image (R,G,B) \\
\hline \multirow{3}{*}{ Blanton Museum } & $\hat{\kappa}$ & $(0.13,-0.07,-0.19)$ & $(0.12,0.12,0.12)$ \\
\cline { 2 - 4 } & $\hat{\sigma}$ & $(17.97,12.57,10.12)$ & $(48.34,48.42,48.53)$ \\
\cline { 2 - 4 } & $\hat{\mu}^{\prime}$ & $(34.35,23.06,16.87)$ & $(74.82,75.26,75.86)$ \\
\hline \multirow{3}{*}{ Sea } & $\hat{\kappa}$ & $(0.06,0.12,0.34)$ & $(0.07,0.12,0.23)$ \\
\cline { 2 - 4 } & $\hat{\sigma}$ & $(36.06,38.74,41.13)$ & $(43.34,46.6,48.32)$ \\
\cline { 2 - 4 } & $\hat{\mu}^{\prime}$ & $(83.87,88.42,82.07)$ & $(73.89,78.67,74.83)$ \\
\hline \multirow{3}{*}{ Forest } & $\hat{\kappa}$ & $(-0.66,-0.64,-0.73)$ & $(0.03,0.06,-0.01)$ \\
\cline { 2 - 4 } & $\hat{\sigma}$ & $(7.55,7.42,4.82)$ & $(45.44,46.5,29.11)$ \\
\cline { 2 - 4 } & $\hat{\mu}^{\prime}$ & $(7.45,7.12,3.64)$ & $(73.42,78.36,79.24)$ \\
\hline
\end{tabular}


Fig. 1(c) illustrates comparisons of the transfer functions (solid lines) from each color band, including the luminance (L) with their CDF of the input images (dash lines). The right picture of Fig. 1(a) shows the image results of the proposed algorithm that could adjust the mean values of the R, G, and B bands to 97.72, 102.04, and 94.87, respectively. Other results would be shown in the experimental results.
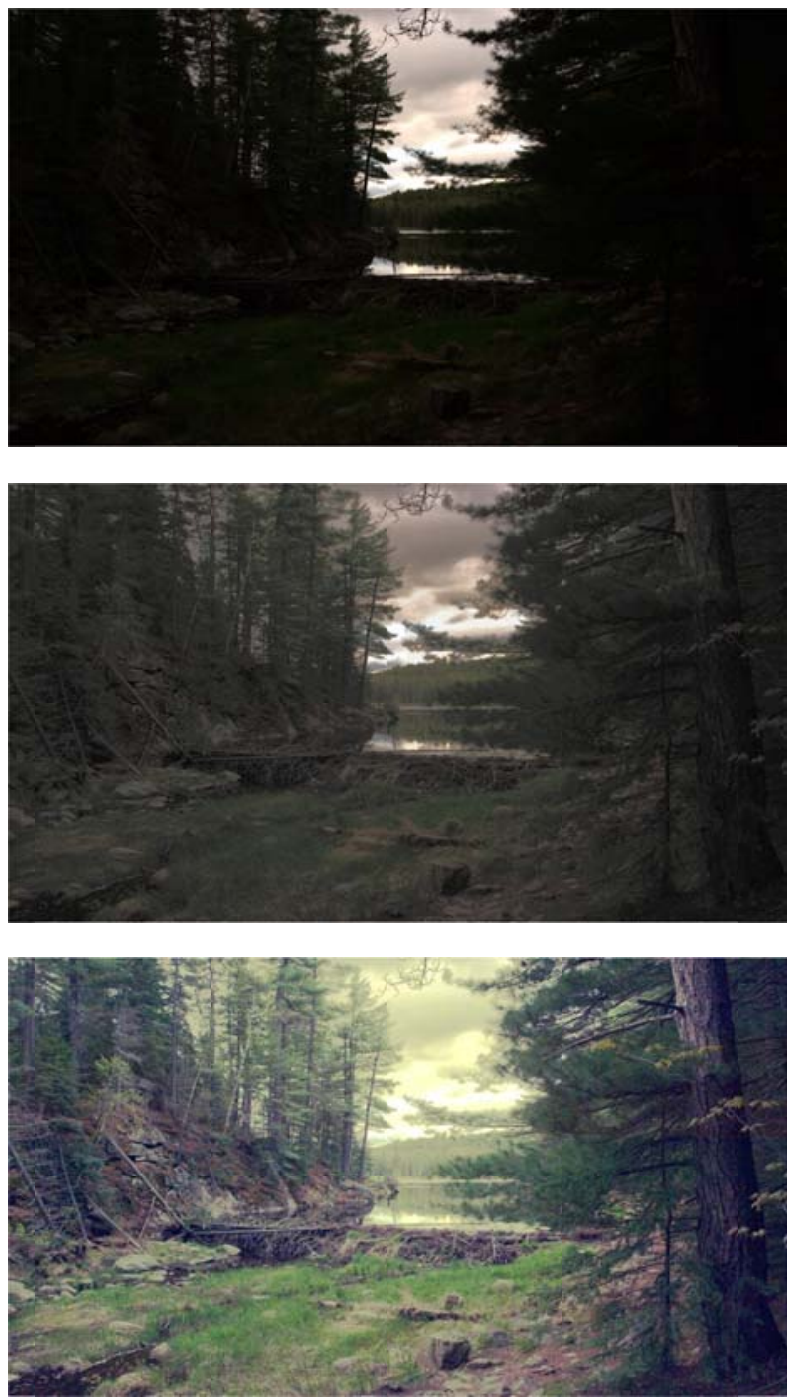

Fig. 5. Forest image enhancement, input, VCEA, and the proposed method (from top to bottom).

\section{Experimental Results}

Our algorithm was tested with low and high dynamic range color images ${ }^{(15,16)}$ that consist of Blanton Museum, Sea, and Forest images. The proposed results were compared with visual contrast enhancement algorithm $(\mathrm{VCEA})^{(10)}$. Tone mapped image quality index (TMQI) ${ }^{(19)}$ with the structure similarity and naturalness measurement were provided for quantitative evaluations.

Table 2. TMQI comparisons.

\begin{tabular}{|l|l|c|c|c|}
\hline Figures & Methods & $\begin{array}{l}\text { Structural } \\
\text { Fidelity }\end{array}$ & $\begin{array}{l}\text { Statistical } \\
\text { Naturalness }\end{array}$ & $\begin{array}{l}\text { Quality } \\
\text { Index }\end{array}$ \\
\hline \multirow{2}{*}{$\begin{array}{l}\text { Blanton } \\
\text { Museum }\end{array}$} & VCEA & 0.9147 & 0.4709 & 0.8963 \\
\cline { 2 - 5 } & HM & 0.9812 & 0.4206 & 0.9042 \\
\hline \multirow{2}{*}{ Sea } & VCEA & 0.9478 & 0.0335 & 0.8061 \\
\cline { 2 - 5 } & HM & 0.9919 & 0.7444 & 0.9605 \\
\hline \multirow{2}{*}{ Forest } & VCEA & 0.9549 & 0.4125 & 0.8961 \\
\cline { 2 - 5 } & HM & 0.9627 & 0.8701 & 0.9721 \\
\hline
\end{tabular}

Our proposed algorithm provides a good visual contrast on the detail area. It is causes from adjusting the color balance and tone tuning by GEV parameters as illustrated in Fig. 3-5. GEV parameters were estimated as seen in the third column of Table 1, and the tuned parameters in the fourth column that were provided to adjust the brightness, contrast, and color balance as shown in the enhanced results in the third row of Fig. 3-5. TMQI comparison results were illustrated in Table 2.

\section{Conclusion}

The experiment results were shown that the enhanced color images by using HM with the transfer function designed with GEV parameters could be provided a good visual contrast image. Enhanced images were adjusted by the location parameters of each color band of the 8 bit samples to replace the peaks of the brightness values for red, green, and blue. The scale parameter could be used to spread the intensity values over the dynamic ranges. The shape parameter was employed to tune the tone of each color band. All image results from the datasets were enhanced according to D55 white point and provided a better image contrast than the input images.

\section{References}

(1) R. Gonzalez and E. R. Woods, "Digital Image Processing," in Digital Image Processing, Pearson Prentice Hall, 2009.

(2) W. K. Pratt, Digital image processing, $4^{\text {th }}$ ed., John Wiley \& Sons, Inc., 2007.

(3) A. K. Jain, Fundamentals of digital image processing, Prentice-Hall, Inc., 1989.

(4) Y. Kim, "Contrast enhancement using brightness preserving 
bi-histogram equalization," IEEE Trans. Consum. Electron, vol. 43, no. 1, p. 1-8, 1997.

(5) K. Wongsritong, K. Kittayaruasiriwat, F. Cheevasuvit, K. Dejhan and A. Somboonkaew, "Contrast enhancement using multipeak histogram equalization with brightness preserving," in Proceedings of the 1998 IEEE Asia-Pacific Conference Circuits and Systems, Chiangmai, Thailand, pp. 455-458, 24-27 November 1998.

(6) S. Chen and A. Ramli, "Contrast enhancement using recursive mean-separate histogram equalization for scalable brightness preservation," IEEE Trans. Consum. Electron., vol. 49, p. 1301-1309, 2003.

(7) C. Wang and Z. Ye, "Brightness preserving histogram equalization with maximum entropy: A variational perspective," IEEE Trans. Consum. Electron., vol. 51, no. 4, p. 1326-1334, 2005.

(8) H. Ibrahim and N. Kong, "Brightness preserving dynamic histogram equalization for image contrast enhancement," IEEE Trans. Consum. Electron., vol. 53, p. 1752-1758, 2007.

(9) D. Sheet, H. Garud, A. Suveer, M. Mahadevappa and J. Chatterjee, "Brightness Preserving Dynamic Fuzzy Histogram Equalization," IEEE Trans. Consum. Electron., vol. 56, no. 4, pp. 2475-2480, 2010.

(10) C.-C. Ting, B.-F. Wu, M.-L. Chung, C.-C. Chiu and Y.-C. $\mathrm{Wu}$, "Visual Contrast Enhancement Algorithm Based on Histogram Equalization," Sensors, vol. 15, pp. 16981-16999, 2015.

(11) S. Kotz and S. Nadarajah, Extreme values distributions: theory and applications, Covent Garden, London: Imperial College Press, 2000.

(12) J. R. M. Hosking, J. R. Wallis and E. F. Wood, "Estimation of the generalized extreme-value distribution by the method of probability weighted moments," Technometrics, vol. 27, no. 3, p. 251-261, 1985.

(13) S. Intajag, S. Kansomkeat and P. Bhurayanontachai, "Histogram specification with generalised extreme value distribution to enhance retinal images," Electronics Letters, vol. 52, no. 8, pp. 596 - 598, 2016.

(14) S. Kullback and R. A. Leibler, "On information and sufficiency," Ann. Math. Stat., vol. 22, no. 1, p. 79-86, 1951.

(15) R. Franzen, " Kodak Lossless True Color Image Suite," 27 January 2013. [Online]. Available: http://r0k.us/graphics/kodak/. [Accessed 10 June 2016].

(16) D. Kundu, D. Ghadiyaram, A. C. Bovik and B. L. Evan,
"ESPL-LIVE HDR Image Quality Database," Embedded Signal Processing Laboratory, 25 May 2016. [Online]. Available:

http://signal.ece.utexas.edu/ debarati/ESPL_LIVE_HDR_D atabase/index.html. [Accessed 10 June 2016].

(17) "Rhian Davis Photography," 19 June 2013. [Online]. Available: http://www.rhian.me/2013_06_01_archive.html. [Accessed 22 June 2016].

(18) G. W. Larson, H. Rushmeier and C. Piatko, "A Visibility Matching Tone Reproduction Operator for High Dynamic Range Scenes," IEEE Transactions on Visualization and Computer Graphics, vol. 3, no. 4, pp. 291-306, 1997.

(19) H. Yeganeh and Z. Wang, "Objective Quality Assessment of Tone-Mapped Images," IEEE Trans. on Image Processing, vol. 22, no. 2, p. $657-667,2013$. 